\title{
Quaternion Discrete Tchebichef Moments and Their Applications
}

\author{
Hongqing Zhu, Qian Li and Qian Liu \\ Department of Electronics and Communications Engineering, East China University \\ of Science and Technology, Shanghai 200237, China \\ hqzhu@ecust.edu.cn
}

\begin{abstract}
The concept of the quaternion is useful for colour image processing and recognition. This paper introduces quaternion discrete Tchebichef moments (QTM), which use the traditional Tchebichef moments (TM) of each colour channel to describe colour images. A set of invariants that are invariant to translation and scale transformations is introduced for colour object recognition and image classification. A theoretical framework is provided for the recognition of colour face images by combining the proposed quaternion Tchebichef moment functions with the sparse representation classification (SRC) strategy for improving recognition despite partial occlusions. Simulation results on standard colour face databases demonstrate the effectiveness of the proposed algorithm, even when the images include Gaussian or pepper-and-salt noise.
\end{abstract}

Keywords: Sparse representation, colour, quaternion, discrete Tchebichef moments, invariant, recognition

\section{Introduction}

Tchebichef moments are discrete orthogonal moments that were introduced by Mukundan [1]. Tchebichef moments have quickly gained popularity as pattern features in image processing [2] and pattern recognition [3] because of their excellent properties. Their implementation does not require numerical approximation because the basis set is discrete in the domain of the discretised image coordinate space. The orthogonality of the moments reduces the redundancy in the representation because the contribution of each Tchebichef moment is independent. Tchebichef moments have been proven to have better descriptive capabilities and invariant properties [4-6] than other moment functions such as Legendre moments [7], Zernike moments [7], pseudoZernike moments [8], and Fourier-Mellin moments [9]. However, Tchebichef moments have been used primarily for processing binary or greyscale images, not colour images, although colour information has been proven to be very useful in pattern recognition. The conventional approach for computing the moment functions and their invariants is to process each colour channel of the colour image separately using greyscale techniques and then to the sum the three individual responses to derive the overall result [10]. Recently, a sophisticated quaternion-based image processing approach was introduced by Sangwine [11] that treats colour images as vector fields by encoding the channel components in the imaginary parts of hypercomplex numbers. This approach has attracted increasing theoretical interest [12] and has been applied in areas such as texture analysis [13], edge detection [14], adaptive filtering [15], and motion estimation [16]. In the present study, we extend the traditional Tchebichef moments to colour 
images using quaternion algebra and discuss the application of Tchebichef moments to image description and invariant pattern recognition.

Invariant pattern recognition is a fundamental tool in machine vision applications. Feature selection, which requires independent geometric transformations, plays a central role in this approach. The original work in this field was presented by Flusser and Suk [17], who proposed moment-based invariant features for image recognition. The invariant properties of moments, which can be utilised as features for object recognition, have received increasing attention [18-19]. However, these methods are mainly limited to processing greyscale images. Several studies have described invariants based on quaternion moments. Recently, Chen et al. [20] used quaternion Zernike moments to construct a set of combined invariants with respect to rotation, scaling, and translation (RST) transformations. The use of quaternion-based FourierMellin moment functions for colour images was investigated by Guo and Zhu [21]. Discrete Tchebichef moments have better performance overall than continuous moments such as Legendre moments, Zernike moments, and Fourier-Mellin moments [22]. The present study extends previous work [3] and constructs a set of translation and scaling invariants using Tchebichef orthogonal polynomials and quaternion algebra for colour image recognition.

Face recognition and face-expression recognition have received considerable attention in pattern recognition because of their wide applications in information security. Recently, Wright et al. [23] introduced sparse-representation-based classification, which efficiently processes data in non-traditional ways. They showed that the choice of features becomes less critical than the number of features when the concept of sparseness is properly used. In their work, the training face images constitute the dictionary, and a test image is classified by finding its sparse representation with respect to this dictionary. This sparse-representation-based classification scheme is successful on standard datasets even when a face is partly occluded. Several methods have recently been developed to improve Wright's approach, including kernel sparse representation [24], Gabor feature-based sparse representation [25], and adaptive sparse representation [26]. However, most of these methods are based on greyscale face images. Because colour face images contain far more information than greyscale face images, recent research has focused on colour face recognition. For example, Rajapakse introduced a non-negative matrix factorisation method to encode the colour channels separately [27]. Wang et al. presented a colour face recognition approach based on the 2DPCA method [28]. However, these schemes do not make use of the spatial relationship information in the image, and their recognition performance is not satisfactory. Sun et al. improved Wang's method by using a quaternion matrix representation scheme to describe the intrinsic colour relationships in images [29]. Inspired by their work, the present study improves upon Wright's method by using discrete Tchebichef moments and quaternion algebra to define the QTM+SRC method. Unlike Wright's method, the proposed method uses quaternions to represent and process colour face images in a holistic framework that not only takes the RGB information into account but also the spatial structure of the image. Unlike common face recognition approaches based on feature extraction, this study uses a colour face recognition scheme based on a sparse representation and Tchebichef moments and treats the recognition problem as one of finding a sparse representation of the test image in terms of the training set. The proposed QTM+SRC method is evaluated using the Face94, Face95, Face96, and Grimace colour face databases, and we also investigate the effectiveness of the approach when the face image is partially occluded. 
The experimental results demonstrate that the QTM+SRC method can achieve better classification accuracy than existing algorithms, with a moderately high computational cost and less complexity.

The rest of this paper is organised as follows. Section 2 briefly reviews quaternions, Tchebichef polynomials and Tchebichef moments. Section 3 defines the quaternion Tchebichef moments. Section 4 introduces the quaternion Tchebichef translation and scale invariants, and we derive the sparse-representation-based and Tchebichefmoment-based colour face recognition methods. Section 5 presents the results of simulations using standard database images and is followed by conclusions and promising future directions in the last section.

\section{Preliminary}

This section reviews some basic definitions and the notation used in the rest of the paper.

\subsection{Quaternion Algebra}

The quaternion, which is a four-dimensional hypercomplex model, was first introduced by British mathematician Hamilton [30] and is denoted by $\mathbf{H}$. A quaternion value $q \in \mathbf{H}$ has a real component and three imaginary components and can be represented in hypercomplex form as

$$
q=a_{0}+a_{1} \boldsymbol{i}+a_{2} \boldsymbol{j}+a_{3} \boldsymbol{k}
$$

where the orthogonal imaginary units satisfy $i j=-j i=k, j k=-k j=i, k i=-i k=j$, and $a_{0}$, $a_{1}, a_{2}, a_{3} \in \mathrm{R} . i, j$, and $k$ are three orthogonal unit vectors defining a frame in 3-D space. They have the property that $i^{2}=j^{2}=k^{2}=-1$. $q$ is a pure quaternion if $a_{0}=0$. The following important basis properties are valid for any two quaternions $q_{1}, q_{2} \in \mathbf{H}$, where $q_{1}=a_{0}+a_{1} \boldsymbol{i}+a_{2} \boldsymbol{j}+a_{3} \boldsymbol{k}$ and $q_{2}=b_{0}+b_{1} \boldsymbol{i}+b_{2} \boldsymbol{j}+b_{3} \boldsymbol{k}$ :

(1) Addition and Subtraction

$$
q_{1} \pm q_{2}=\left(a_{0} \pm b_{0}\right)+\left(a_{1} \pm b_{1}\right) \boldsymbol{i}+\left(a_{2} \pm b_{2}\right) \boldsymbol{j}+\left(a_{3} \pm b_{3}\right) \boldsymbol{k}
$$

(2) Multiplication

$$
\begin{aligned}
q_{1} \cdot q_{2}= & \left(a_{0} b_{0}-a_{1} b_{1}-a_{2} b_{2}-a_{3} b_{3}\right)+\left(a_{0} b_{1}+a_{1} b_{0}+a_{2} b_{3}-a_{3} b_{2}\right) \boldsymbol{i}+\left(a_{0} b_{2}+a_{2} b_{0}+a_{3} b_{1}-a_{1} b_{3}\right) \boldsymbol{j} \\
& +\left(a_{0} b_{3}+a_{3} b_{0}+a_{1} b_{2}-a_{2} b_{1}\right) \boldsymbol{k}
\end{aligned}
$$

(3) Quaternion conjugate

$$
\bar{q}_{1}=a_{0}-a_{1} \boldsymbol{i}-a_{2} \boldsymbol{j}-a_{3} \boldsymbol{k}
$$

(4) Quaternion norm

$$
\left\|q_{1}\right\|=\sqrt{a_{0}^{2}+a_{1}^{2}+a_{2}^{2}+a_{3}^{2}}
$$

Moxey proposed a new model for the quaternion based on the following equation [31]:

$$
q=a_{0}+a_{1} \boldsymbol{i}+a_{2} \boldsymbol{j}+a_{3} \boldsymbol{k}=A+B \boldsymbol{j}
$$

where $A=a_{0}+a_{1} i$ and $B=a_{2}+a_{3} i$. 


\subsection{Tchebichef Orthogonal Moments}

The Tchebichef polynomials of order $n$ are defined as [1]

$$
t_{n}(x)=n ! \sum_{k=0}^{n}(-1)^{n-k}\left(\begin{array}{c}
N-1-k \\
n-k
\end{array}\right)\left(\begin{array}{c}
n+k \\
n
\end{array}\right)\left(\begin{array}{l}
x \\
k
\end{array}\right)
$$

They satisfy the following orthogonality property in the discrete domain:

$$
\sum_{x=0}^{N-1} t_{n}(x) t_{m}(x)=\rho(n, N) \delta_{n m}
$$

where $\delta_{n m}$ is the Kronecker function, i.e., $\delta_{n m}=1$ if $p=q$ and 0 otherwise, and the squared norm $\rho(n, N)$ is given by

$$
\rho(n, N)=\frac{(N+p) !}{(2 p+1)(N-p-1) !}
$$

Tchebichef polynomials satisfy the following recurrence relation

$$
(n+1) t_{n+1}(x)-(2 n+1)(2 x-N+1) t_{n}(x)+n\left(N^{2}-n^{2}\right) t_{n-1}(x)=0, \quad n=1,2, \ldots, N-1
$$

This set of polynomials is not suitable for defining moments; the scaled discrete Tchebichef polynomials are defined as

$$
\tilde{t}_{n}(x)=\frac{t_{n}(x)}{\beta(n, N)}
$$

where $\beta(n, N)$ is a suitable constant that is independent of $x$. A particular choice of $\beta(n, N)$ is

$$
\beta(n, N)=\sqrt{\rho(n, N)}
$$

Mukundan [1] defined the 2-D discrete Tchebichef moment of order $(p+q)$ of an image $f$ $(x, y)$ with size $N \times N$ as

$$
T_{p q}=\sum_{x=0}^{N-1} \sum_{y=0}^{N-1} \tilde{t}_{p}(x) \tilde{t}_{q}(y) f(x, y)
$$

Eq. (13) leads to the following exact image reconstruction (i.e., the inverse moment transform):

$$
\hat{f}(x, y)=\sum_{p=0}^{N-1} \sum_{q=0}^{N-1} \tilde{t}_{p}(x) \tilde{t}_{q}(y) T_{p q}
$$

\section{Colour Image Analysis using Quaternion Tchebichef Moments}

Inspired by quaternion Fourier transforms and quaternion cosine transforms of 2-D functions [32-33], this study defines quaternion (or hypercomplex) Tchebichef moments, which are a generalisation of traditional Tchebichef moments.

Without loss of generality, we consider here only RGB colour images. The generalisation to other types of colour images is not difficult. Let $f(x, y)$ be an RGB image; the three channels of the image can be represented using the three components of a pure quaternion as follows:

$$
f(x, y)=f_{R}(x, y) \boldsymbol{i}+f_{G}(x, y) \boldsymbol{j}+f_{B}(x, y) \boldsymbol{k}
$$


where $f_{R}(x, y), f_{\mathrm{G}}(x, y)$ and $f_{B}(x, y)$ represent the red, green and blue components of the colour image, respectively. This representation effectively equates the RGB colour cube to the right-handed coordinate frame imposed by the imaginary part of the quaternion space and treats the colour image as a whole, rather than as separate components. We now introduce the definition of the right-side form of the quaternion Tchebichef moments of a function of two variables based on Ell's formula [32].

Definition 1: Given a quaternion root of $-1, \boldsymbol{\mu}$, for an $N \times N$ colour image $f(x, y)$, the right-side form of the forward quaternion Tchebichef moments of the image is defined as

$$
\operatorname{QTM}_{p q}^{R}(f)=\sum_{x=0}^{N-1} \sum_{y=0}^{N-1}\left(f_{R}(x, y) \boldsymbol{i}+f_{G}(x, y) \boldsymbol{j}+f_{B}(x, y) \boldsymbol{k}\right) \tilde{t}_{p}(x) \tilde{t}_{q}(y) \boldsymbol{\mu}
$$

where $\boldsymbol{\mu}=-(\boldsymbol{i}+\boldsymbol{j}+\boldsymbol{k}) / \sqrt{3}$ and $\boldsymbol{\mu}^{2}=\boldsymbol{i}^{2}=\boldsymbol{j}^{2}=\boldsymbol{k}^{2}=-1$. This choice corresponds to the grey line in RGB space, on which all three components are equal.

$$
\begin{aligned}
\operatorname{QTM}_{p q}^{R}(f)= & -\sum_{x=0}^{N-1} \sum_{y=0}^{N-1}\left(f_{R}(x, y) \boldsymbol{i}+f_{G}(x, y) \boldsymbol{j}+f_{B}(x, y) \boldsymbol{k}\right) \tilde{t}_{p}(x) \tilde{t}_{q}(y)(\boldsymbol{i}+\boldsymbol{j}+\boldsymbol{k}) / \sqrt{3} \\
= & \frac{1}{\sqrt{3}}\left[\sum_{x=0}^{N-1} \sum_{y=0}^{N-1}\left(f_{R}(x, y)+f_{G}(x, y)+f_{B}(x, y)\right) \tilde{t}_{p}(x) \tilde{t}_{q}(y)\right] \\
& -\frac{1}{\sqrt{3}} i\left[\sum_{x=0}^{N-1} \sum_{y=0}^{N-1}\left(f_{G}(x, y)-f_{B}(x, y)\right) \tilde{t}_{p}(x) \tilde{t}_{q}(y)\right] \\
& -\frac{1}{\sqrt{3}} j\left[\sum_{x=0}^{N-1} \sum_{y=0}^{N-1}\left(f_{B}(x, y)-f_{R}(x, y)\right) \tilde{t}_{p}(x) \tilde{t}_{q}(y)\right] \\
& -\frac{1}{\sqrt{3}} \boldsymbol{k}\left[\sum_{x=0}^{N-1} \sum_{y=0}^{N-1}\left(f_{R}(x, y)-f_{G}(x, y)\right) \tilde{t}_{p}(x) \tilde{t}_{q}(y)\right] \\
& \operatorname{QTM}_{p q}^{R}(f)=A_{0}+\boldsymbol{i} A_{1}+\boldsymbol{j} A_{2}+\boldsymbol{k} A_{3}
\end{aligned}
$$

where

$$
\begin{gathered}
A_{0}=\frac{1}{\sqrt{3}}\left[\mathrm{TM}_{\mathrm{R}}+\mathrm{TM}_{\mathrm{G}}+\mathrm{TM}_{\mathrm{B}}\right], A_{1}=-\frac{1}{\sqrt{3}}\left[\mathrm{TM}_{\mathrm{G}}-\mathrm{TM}_{\mathrm{B}}\right], \\
A_{2}=-\frac{1}{\sqrt{3}}\left[\mathrm{TM}_{\mathrm{B}}-\mathrm{TM}_{\mathrm{R}}\right], A_{3}=-\frac{1}{\sqrt{3}}\left[\mathrm{TM}_{\mathrm{R}}-\mathrm{TM}_{\mathrm{G}}\right]
\end{gathered}
$$

$\mathrm{TM}_{\mathrm{R}}, \mathrm{TM}_{\mathrm{G}}$, and $\mathrm{TM}_{\mathrm{B}}$ are the traditional discrete Tchebichef moments for the red, green, and blue components, respectively, of the colour image. This paper defines the left-side forward quaternion Tchebichef moments similarly by interchanging the positions of the colour image and the imaginary part $\boldsymbol{\mu}$.

Definition 2: Given a quaternion root of $-1, \boldsymbol{\mu}$, for an $N \times N$ colour image $f(x, y)$, the leftside form of the forward Quaternion Tchebichef moments of the image is defined as

$$
\operatorname{QTM}_{p q}^{L}(f)=\sum_{x=0}^{N-1} \sum_{y=0}^{N-1} \boldsymbol{\mu}\left(f_{R}(x, y) \boldsymbol{i}+f_{G}(x, y) \boldsymbol{j}+f_{B}(x, y) \boldsymbol{k}\right) \tilde{t}_{p}(x) \tilde{t}_{q}(y)
$$


Corresponding to the QTM, there are two forms of the inverse quaternion discrete Tchebichef moments (IQTM), which satisfy the following equations.

$$
\begin{gathered}
\operatorname{IQTM}_{p q}^{R}(f)=\sum_{p=0}^{N-1} \sum_{q=0}^{N-1}\left(A_{0}+\boldsymbol{i} A_{1}+\boldsymbol{j} A_{2}+\boldsymbol{k} A_{3}\right) \tilde{t}_{p}(x) \tilde{t}_{q}(y) \boldsymbol{\mu} \\
\operatorname{IQTM}_{p q}^{R}(f)=-\sum_{p=0}^{N-1} \sum_{q=0}^{N-1}\left(A_{0}+\boldsymbol{i} A_{1}+\boldsymbol{j} A_{2}+\boldsymbol{k} A_{3}\right) \tilde{t}_{p}(x) \tilde{t}_{q}(y)(\boldsymbol{i}+\boldsymbol{j}+\boldsymbol{k}) / \sqrt{3} \\
=\frac{1}{\sqrt{3}}\left[\sum_{p=0}^{N-1} \sum_{q=0}^{N-1}\left(A_{1}+A_{2}+A_{3}\right) \tilde{t}_{p}(x) \tilde{t}_{q}(y)\right]-\frac{1}{\sqrt{3}} i\left[\sum_{p=0}^{N-1} \sum_{q=0}^{N-1}\left(A_{0}+A_{2}-A_{3}\right) \tilde{t}_{p}(x) \tilde{t}_{q}(y)\right] \\
\\
\frac{1}{\sqrt{3}} j\left[\sum_{p=0}^{N-1} \sum_{q=0}^{N-1}\left(A_{0}-A_{1}+A_{3}\right) \tilde{t}_{p}(x) \tilde{t}_{q}(y)\right]-\frac{1}{\sqrt{3}} \boldsymbol{k}\left[\sum_{p=0}^{N-1} \sum_{q=0}^{N-1}\left(A_{0}+A_{1}-A_{2}\right) \tilde{t}_{p}(x) \tilde{t}_{q}(y)\right] \\
=a_{0}+\boldsymbol{i} a_{1}+j a_{2}+\boldsymbol{k} a_{3} \\
a_{0}=\frac{1}{\sqrt{3}}\left[\operatorname{ITM}\left(A_{1}\right)+\operatorname{ITM}\left(A_{2}\right)+\operatorname{ITM}\left(A_{3}\right)\right], a_{1}=-\frac{1}{\sqrt{3}} \boldsymbol{i}\left[\operatorname{ITM}\left(A_{0}\right)+\operatorname{ITM}\left(A_{2}\right)-\operatorname{ITM}\left(A_{3}\right)\right], \\
a_{2}=-\frac{1}{\sqrt{3}} j\left[\operatorname{ITM}\left(A_{0}\right)-\operatorname{ITM}\left(A_{1}\right)+\operatorname{ITM}\left(A_{3}\right)\right], a_{3}=-\frac{1}{\sqrt{3}} \boldsymbol{k}\left[\operatorname{ITM}\left(A_{0}\right)+\operatorname{ITM}\left(A_{1}\right)-\operatorname{ITM}\left(A_{2}\right)\right]
\end{gathered}
$$

where $a_{1}, a_{2}$, and $a_{3}$ are the three colour components of the reconstructed image. The left-side inverse transform is similarly defined as follows:

$$
\operatorname{IQTM}_{p q}^{L}(f)=\sum_{p=0}^{N-1} \sum_{q=0}^{N-1} \boldsymbol{\mu}\left(A_{0}-\boldsymbol{i} A_{1}-\boldsymbol{j} A_{2}-\boldsymbol{k} A_{3}\right) \tilde{t}_{p}(x) \tilde{t}_{q}(y)
$$

This study will use the right-side forms $\operatorname{QTM}_{p q}^{R}(f)$ and $\operatorname{IQTM}_{p q}^{R}(f)$ as the default QTM and IQTM, respectively, unless stated otherwise. The QTM pair is separable, and thus may be evaluated with a $1 \mathrm{D}$ summation over the rows and columns of the input array.

\section{Colour Image Object Recognition using Quaternion Tchebichef Moments}

\subsection{Quaternion Tchebichef Translation Invariants}

The 2-D quaternion Tchebichef translation invariants can be obtained by evaluating their central moments, which are defined by

$$
Q T_{n m}^{\prime}=\sum_{x=0}^{N-1} \sum_{y=0}^{N-1} \tilde{t}_{n}\left(x-x_{0}\right) \tilde{t}_{m}\left(y-y_{0}\right)\left(f_{R}(x, y) \boldsymbol{i}+f_{G}(x, y) \boldsymbol{j}+f_{B}(x, y) \boldsymbol{k}\right)(\boldsymbol{i}+\boldsymbol{j}+\boldsymbol{k}) / \sqrt{3}
$$

where $\left(x_{0}, y_{0}\right)$ denotes the image centroid coordinates. As shown in our previous work [3], the translated Tchebichef polynomials in the $x$ direction can be derived as follows:

$$
\tilde{t}_{n}\left(x-x_{0}\right)=\sum_{k=0}^{n} \tilde{v}_{n(n-k)} \tilde{t}_{n-k}(x)
$$


where

$$
\tilde{v}_{n(n-k)}=\frac{1}{B_{(n-k)(n-k)}}\left[\sum_{l=0}^{k}\left(\begin{array}{c}
n-k+l \\
l
\end{array}\right) B_{n(n-k+l)}<-x_{0}>-\sum_{i=0}^{k-1} B_{(n-i)(n-k)} \tilde{v}_{n(n-i)}\right]
$$

with

$$
B_{n k}=\frac{(n+k) !}{\sqrt{\rho(n, N)(n-k) !(k !)^{2}}}<n-N>_{n-k}
$$

The translated Tchebichef polynomials in the $y$ direction can be obtained using the same procedure.

\subsection{Quaternion Tchebichef Scale Invariants}

Assume that the scale factors for the original image are $a$ and $b$ in the $x$ direction and the $y$ direction, respectively. The scaled quaternion Tchebichef moments can be defined as follows:

$$
Q T_{n m}^{\prime \prime}=\sum_{k=0}^{n} \sum_{l=0}^{m} \lambda_{n k} \lambda_{m l} \tilde{t}_{k}(a x) \tilde{t}_{l}(b y)\left(f_{R}(x, y) \boldsymbol{i}+f_{G}(x, y) \boldsymbol{j}+f_{B}(x, y) \boldsymbol{k}\right)(\boldsymbol{i}+\boldsymbol{j}+\boldsymbol{k}) / \sqrt{3}
$$

Using the Stirling numbers of the first kind, $s(k, i)$, the scaled Tchebichef polynomials in the $x$ direction can be written as

$$
\tilde{t}_{n}(x)=\sum_{i=0}^{n} C(n, i) x^{i}, \tilde{t}_{n}(a x)=\sum_{i=0}^{n} C(n, i) a^{i} x^{i}
$$

where

$$
C(n, i)=\sum_{k=0}^{n-i} \tilde{B}_{n, n-k} s(n-k, i)
$$

Eq. (27) can be rewritten as

$$
Q T_{n m}^{\prime \prime}=a^{n+1} b^{m+1} \sum_{k=0}^{n} \sum_{l=0}^{m} \lambda_{n k} \lambda_{m l} \tilde{t}_{k}(x) \tilde{t}_{l}(y)\left(f_{R}(x, y) \boldsymbol{i}+f_{G}(x, y) \boldsymbol{j}+f_{B}(x, y) \boldsymbol{k}\right)(\boldsymbol{i}+\boldsymbol{j}+\boldsymbol{k}) / \sqrt{3}
$$

The relationship between the original and scaled Tchebichef moments can then be established as

$$
Q \varphi_{n m}=\sum_{k=0}^{n} \sum_{l=0}^{m} \lambda_{n k} \lambda_{m l} Q T_{k l}^{\prime \prime}=a^{n+1} b^{m+1} \sum_{k=0}^{n} \sum_{l=0}^{m} \lambda_{n k} \lambda_{m l} Q T_{k l}
$$

where

$$
\lambda_{n k}=\sum_{r=0}^{n-k-1} \frac{-C_{(n-r) k} \lambda_{n(n-r)}}{C_{k k}}, \quad 0 \leq k \leq n
$$

To obtain the scale invariants, the scale factors $a$ and $b$ must be eliminated. The following constructed invariants are not influenced by the scale factors. 


$$
Q \psi_{n m}=\frac{Q \varphi_{n m} Q \varphi_{00}^{r+1}}{Q \varphi_{n+r, 0} Q \varphi_{0, m+r}}, \quad n, m=1,2, \ldots \quad r=1,2,3 \ldots
$$

The combined translation and scale invariants are computed using Eq. (33), where the $Q \varphi_{n m}$ on the right-hand side of Eq. (33) are computed using Eqs. (23) and (31).

\subsection{Face Recognition based on Quaternion Tchebichef Moments}

For an $N_{1} \times N_{2}$ image $f(x, y)$, one can resize all the pixels using a column v. Any test sample $y$ of this object class can then be approximately represented in the linear span of the training class.

$$
\mathbf{A}=\left[\mathbf{v}_{1,1}, \mathbf{v}_{1,2}, \cdots, \mathbf{v}_{1, s_{1}}, \cdots, \mathbf{v}_{i, 1}, \mathbf{v}_{i, 2}, \cdots, \mathbf{v}_{i, s_{i}}, \cdots, \mathbf{v}_{k, 1}, \mathbf{v}_{k, 2}, \cdots, \mathbf{v}_{k, s_{k}}\right]
$$

where $\mathbf{v}_{i, j}$ is the $i$ th object class of the $j$ th picture, $s_{i}$ is the number of pictures of the $i$ th object class, and $S$ is the total number of pictures in the training set. Suppose that test image $y$ is from the $i$ th object; according to Eq. (34), the contribution of the complete training set to this image can be written as

$$
\mathbf{y}=0 \cdot \mathbf{v}_{1,1}+0 \cdot \mathbf{v}_{1,2}+\ldots+a_{i, 1} \mathbf{v}_{i, 1}+a_{i, 2} \mathbf{v}_{i, 2}+\ldots+a_{i, s_{i}} \mathbf{v}_{i, s_{i}}+\ldots+0 \cdot \mathbf{v}_{k, 1}+0 \cdot \mathbf{v}_{k, 2}+\ldots+0 \cdot \mathbf{v}_{k, s_{k}}
$$

The $i$ th training set provides the entire contribution to this test image; the linear spans of the other object training sets cannot describe the image, and thus they provide no contributions to the image. The linear combination of $\mathbf{y}$ can then be written in matrix form:

$$
\mathbf{y}=\mathbf{A} \mathbf{x}
$$

where $\mathbf{x}=\left[0, \cdots, 0, a_{i, 1}, a_{i, 2}, \cdots, a_{i, s_{i}}, 0, \cdots, 0\right]^{T} \in R^{S}$. When the database is sufficient, the $S$ dimensional vector $\mathbf{x}$ has only $s_{i}$ non-zero values, where $s_{i} \square S$; therefore, the representation of Eq. (35) is sparse. One can rewrite the traditional Tchebichef moments in vector form

$$
\begin{aligned}
T_{n m} & =\left[\tilde{t}_{n}(0) \tilde{t}_{m}(0), \cdots, \tilde{t}_{n}(0) \tilde{t}_{m}\left(N_{2}-1\right), \tilde{t}_{n}(1) \tilde{t}_{m}(0), \cdots, \tilde{t}_{n}\left(N_{1}-1\right) \tilde{t}_{m}\left(N_{2}-1\right)\right]\left[\begin{array}{c}
f(0,0) \\
\vdots \\
f\left(0, N_{2}-1\right) \\
\vdots \\
f\left(N_{1}-1, N_{2}-1\right)
\end{array}\right] \\
& =\tilde{\mathbf{t}}_{\mathbf{n m}} \mathbf{v}
\end{aligned}
$$

where $\mathbf{v} \in R^{l}$ is an image $f(x, y)$ in column order, and the image size $l=N_{1} \times N_{2}$. According to Eq. (37), one has

$$
\tilde{\mathbf{A}}=\left[\begin{array}{cccccc}
T_{00}^{(1)} & T_{00}^{(2)} & \cdots & T_{00}^{\left(s_{i}\right)} & \cdots & T_{00}^{\left(s_{k}\right)} \\
T_{01}^{(1)} & T_{01}^{(2)} & \cdots & T_{01}^{\left(s_{i}\right)} & \cdots & T_{01}^{\left(s_{k}\right)} \\
\vdots & \vdots & \vdots & \vdots & \vdots & \vdots \\
T_{p q}^{(1)} & T_{p q}^{(2)} & \cdots & T_{p q}^{\left(s_{i}\right)} & \cdots & T_{p q}^{\left(s_{k}\right)}
\end{array}\right]=\mathbf{M A}
$$


where $p+q=L$. In the current study, the Tchebichef moments $\mathbf{M}$ are substituted for the random matrix in the SRC algorithm [22]. M can be defined as

$$
\mathbf{M}=\left(\tilde{\mathbf{t}}_{\mathbf{0 0}}, \tilde{\mathbf{t}}_{\mathbf{0} 1}, \cdots, \tilde{\mathbf{t}}_{\mathbf{0 q}}, \tilde{\mathbf{t}}_{\mathbf{1 0}}, \cdots, \tilde{\mathbf{t}}_{\mathbf{p q}}\right)^{T} \in R^{d \times l}
$$

$\mathbf{M}$ is a matrix with size $d \times l(d=(L+1)(L+2) / 2)$. Multiplying Eq. (36) by matrix $\mathbf{M}$ on both sides gives

$$
\tilde{\mathbf{y}}=\mathbf{M y}=\mathbf{M A x}=\tilde{\mathbf{A}} \mathbf{x}
$$

For each colour component $R, G$, and $B$, one can calculate $\tilde{\mathbf{y}}_{r}, \tilde{\mathbf{y}}_{g}$, and $\tilde{\mathbf{y}}_{b}$. The quaternion sparse representation $\tilde{\mathbf{y}}$ can thus be derived as

$$
\hat{\mathbf{y}}=\operatorname{QTM}(\tilde{\mathbf{y}})
$$

The recognition problem for an image $\mathbf{y}$ can be obtained by solving the following $l 1$-norm problem:

$$
\arg \min \|\mathbf{x}\|_{1}, \quad \text { s.t. } \quad \hat{\mathbf{y}}=\operatorname{QTM}(\tilde{\mathbf{y}})=\operatorname{QTM}(\tilde{\mathbf{A}} \mathbf{x})
$$

In this study, the Tchebichef polynomials are not related to unknown images and thus can be calculated and saved ahead. The following algorithm summarises the complete recognition procedure:

(1) The inputs are a matrix of training samples containing $S$ images of $k$ objects and a test sample.

(2) Calculate the quaternion representation for each training set $\tilde{\mathbf{A}}$ as $\hat{\mathbf{A}}$, and calculate the quaternion representation for test image $\tilde{\mathbf{y}}$ as $\hat{\mathbf{y}}$. Normalise the columns of $\hat{\mathbf{A}}$ and $\hat{\mathbf{y}}$.

(3)Compute the residual $r_{i}(\hat{\mathbf{y}})=\left\|\mathbf{y}-\hat{\mathbf{A}} \delta_{i}(\mathbf{x})\right\|_{2}$ for $i=1,2, \ldots, k$, where $\delta_{i}(\mathbf{x})$ denotes the component of object $i$ in $\mathbf{x}$; the other components are zeros.

(4)The output of the algorithm is identity $(\mathbf{y})=\arg \min r_{i}(\hat{\mathbf{y}}=\mathrm{QTM}(\mathbf{M y}))$.

\section{Experimental Results}

\subsection{Invariance Tests}

In this subsection, two experiments are performed to illustrate the invariance properties of the proposed QTM method. The first experiment aims to verify the performance of the invariants under translation and scaling. The second experiment verifies the classification capability of the QTM features. We choose the database used in Chen et al. [20] to compare our results. This database consists of 7200 images evenly divided into 100 categories. Ten images are randomly selected for the training set (Figure 1). The test set is generated by translating the images by $\Delta x, \Delta y=-10,-3,4,11$ in the horizontal and vertical directions and by scaling with a scaling factor $\lambda \in\{0.5$, $0.8,1.1,1.4\}$, forming a set of 2560 images from the 10 original images. A bilinear interpolation is used when required. Two $128 \times 128$ images are randomly chosen to evaluate the proposed QTM invariants. The invariant values, computed using Eq. (33), are listed in Tables 1 and 2. In this study, the absolute value of the logarithm of the invariant is used to reduce the dynamic range. Tables 1 and 2 show that the proposed 
invariants remain invariant as the translation and scaling is varied. The second experiment compares the classification accuracy of the QTM and the QZM [20]. For the recognition task, we use the following feature vector:

$$
V=\left[I_{00}, I_{11}, I_{20}, I_{02}, I_{21}, I_{12}, I_{30}\right]
$$

where $I_{n m}$ is the same as $Q \psi_{n m}$ defined previously. The Euclidean distance is used as the classification measure. Additionally, to test the robustness of the proposed method in a noisy environment, we add Gaussian random noise or pepper-and-salt noise to the test images. Table 3 shows the classification results from the two moment invariants. Table 3 shows that the rate of accurate classification using the QTM is higher in each test than the state-of-art QZM algorithm, proving the feasibility of the proposed method.

\section{Table 1. The QTM Invariants for a Colour Image}

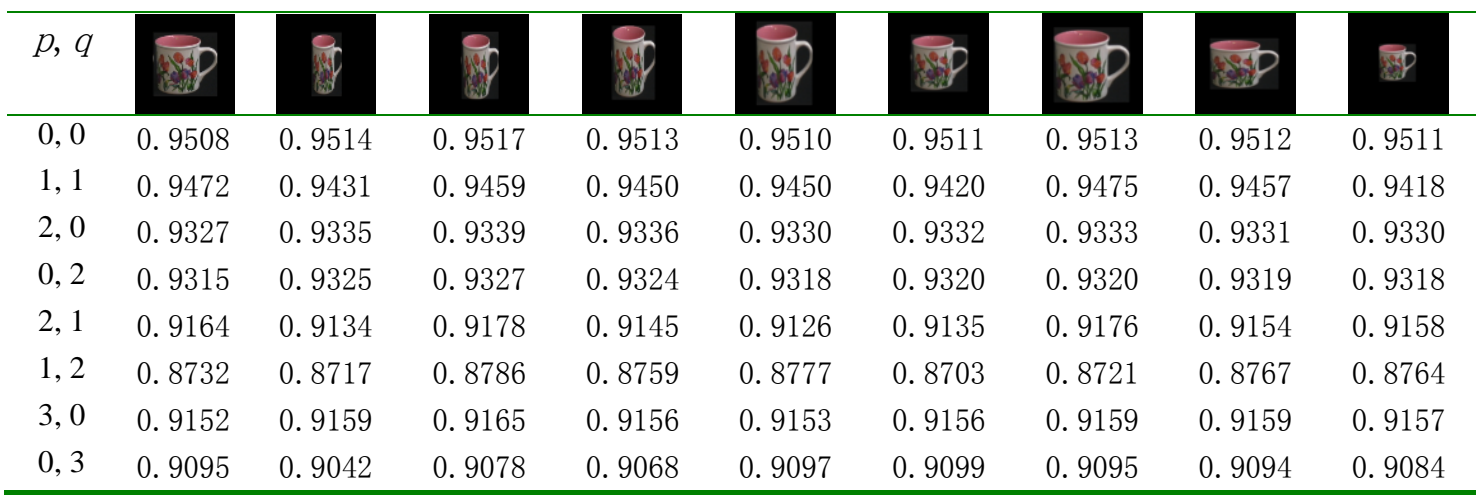

Table 2. The QTM Invariants for a Second Colour Image

\begin{tabular}{|c|c|c|c|c|c|c|c|c|c|}
\hline$p, q$ & 3 & 8 & 8 & 8 & (1) & 8 & 8 & & 3 \\
\hline 0,0 & 0.9438 & 0.9447 & 0.9448 & 0.9443 & 0.9435 & 0.9449 & 0.9443 & 0.9448 & 0.9449 \\
\hline 1,1 & 0.9087 & 0.9011 & 0.9089 & 0.9023 & 0.9030 & 0.9087 & 0.9018 & 0.9088 & 0.9026 \\
\hline 2,0 & 0.9278 & 0.9287 & 0.9282 & 0.9286 & 0.9278 & 0.9290 & 0.9284 & 0.9284 & 0.9288 \\
\hline 0,2 & 0.9170 & 0.9184 & 0.9182 & 0.9177 & 0.9165 & 0.9185 & 0.9176 & 0.9182 & 0.9184 \\
\hline 2,1 & 0.8919 & 0.8918 & 0.8904 & 0.8916 & 0.8909 & 0.8931 & 0.8917 & 0.8984 & 0.8917 \\
\hline 1,2 & 0.8732 & 0.8731 & 0.8750 & 0.8777 & 0.8776 & 0.8717 & 0.8760 & 0.8668 & 0.8793 \\
\hline 3,0 & 0.9129 & 0.9139 & 0.9131 & 0.9135 & 0.9127 & 0.9140 & 0.9135 & 0.9135 & 0.9134 \\
\hline 0,3 & 0.8977 & 0.8969 & 0.8974 & 0.8973 & 0.8967 & 0.8992 & 0.8977 & 0.8992 & 0.8972 \\
\hline
\end{tabular}

Table 3. The Recognition Rates of QZM and QTM with Different Levels of Noise

\begin{tabular}{cccccccc}
\hline \multirow{2}{*}{ Method } & Noise-Free & \multicolumn{3}{c}{ Gaussian Noise (Variance) } & \multicolumn{4}{c}{ Pepper \& Salt Noise } \\
\cline { 3 - 7 } & & 0.01 & 0.02 & $1 \%$ & $2 \%$ & $3 \%$ & $4 \%$ \\
\hline QZM & $100 \%$ & $91.32 \%$ & $77.56 \%$ & $91.18 \%$ & $89.04 \%$ & $82.76 \%$ & $80.53 \%$ \\
QTM & $100 \%$ & $93.75 \%$ & $80.00 \%$ & $93.75 \%$ & $90.63 \%$ & $86.25 \%$ & $84.38 \%$ \\
\hline
\end{tabular}


Table 4. The Effect of Varying the Order $L$ in Colour Face Recognition

\begin{tabular}{cccccc} 
& $L=3$ & $L=6$ & $L=9$ & $L=14$ & $L=30$ \\
& $(d=10)$ & $(d=28)$ & $(d=55)$ & $(d=120)$ & $(d=540)$ \\
\hline Face95 & $91.69 \%$ & $94.44 \%$ & $97.80 \%$ & $98.80 \%$ & $100 \%$ \\
Face96 & $96.58 \%$ & $97.17 \%$ & $97.52 \%$ & $98.10 \%$ & $100 \%$ \\
\hline
\end{tabular}

Table 5. The Recognition Rates of the Different Methods of Colour Face Recognition

\begin{tabular}{cccccc}
\hline Methods & L/d & Face94 & Face95 & Face96 & Grimace \\
\hline Random + SRC & $4 / 15$ & $89.47 \%$ & $60.56 \%$ & $83.94 \%$ & $98.33 \%$ \\
& $5 / 21$ & $89.47 \%$ & $67.92 \%$ & $92.24 \%$ & $99.44 \%$ \\
& $6 / 28$ & $98.51 \%$ & $79.17 \%$ & $95.33 \%$ & $100.00 \%$ \\
\hline Downsample + & $4 / 15$ & $96.67 \%$ & $61.11 \%$ & $89.94 \%$ & $99.44 \%$ \\
SRC & $5 / 21$ & $98.60 \%$ & $67.64 \%$ & $91.56 \%$ & $100.00 \%$ \\
& $6 / 28$ & $98.95 \%$ & $73.61 \%$ & $95.73 \%$ & $100.00 \%$ \\
\hline QTM + SRC & $4 / 15$ & $99.74 \%$ & $92.08 \%$ & $96.64 \%$ & $100.00 \%$ \\
& $5 / 21$ & $99.91 \%$ & $92.92 \%$ & $97.30 \%$ & $100.00 \%$ \\
& $6 / 28$ & $99.91 \%$ & $94.44 \%$ & $97.17 \%$ & $100.00 \%$ \\
\hline
\end{tabular}

\subsection{SRC-based QTM for Colour Face Recognition}

To evaluate the effectiveness of the proposed method, a colour face recognition experiment is performed. The experiments are conducted using the Face94, Face95, Face 96 and Grimace colour face databases assembled by the Computer Vision Group of Essex University [34]. The Face94 database consists of 20 female and 133 male face images from 20 distinct subjects, with variations in the illumination and the facial expression. Some test images from this database are shown in Figure 2(a). Figure 2(b) shows several images from the Face 95 database, which includes 72 subjects. The image background in this database is a red curtain. Background variation is caused by shadows as the subject moves forward. The Grimace database contains 20 images each of 18 male and female subjects. These images have a plain background and very little variation in illumination (see Figure 2(c)). To provide a challenging test, the Face96 database, consisting of over 152 subjects, is included. The images in this database vary in the position of the face in the image, the illumination, the arrangement of the artificial lighting, the facial expression, and the complexity of the background.
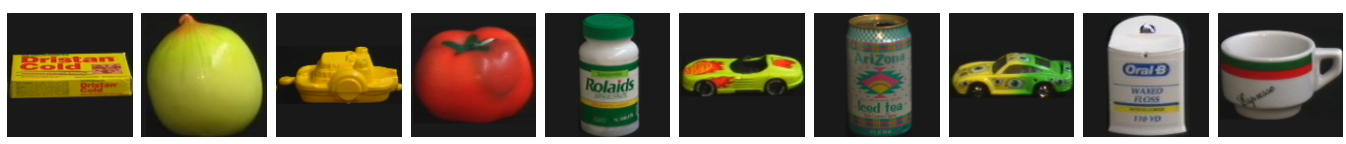

Figure 1. The Test Images used in the Recognition Tasks 

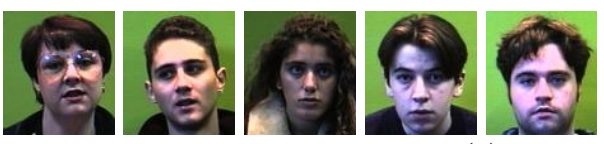

(a)
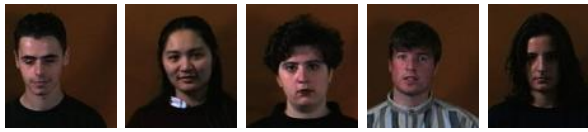

(b)
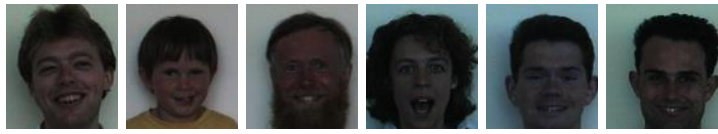

(c)
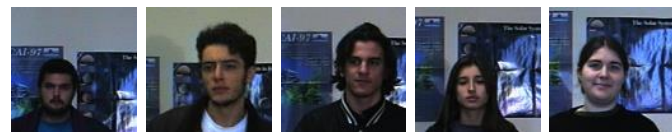

(d)

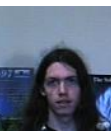

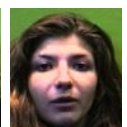
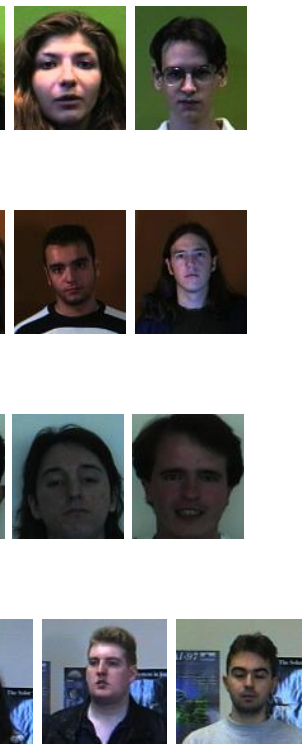

Figure 2. Some of the Test Images used in the Face Recognition Tasks, from (a) the Face94 database, (b) the Face95 database, (c) the Grimace database, and (d) the Face96 database

We test the recognition rate of quaternion Tchebichef moments of order $L$ first using the Face95 and Face96 databases and then using all of the databases. In these experiments, all subjects in these databases are used. For each subject, we randomly choose 10 images as the training data. The remaining images serve as the test data. For each test image, we apply the proposed approach using different moment orders $L$. Table 4 shows the recognition rate as $L$ is varied. The QTM+SRC method performs very well, and a higher $L$ leads to a higher recognition rate. However, when the order of the orthogonal Tchebichef moments is high, the computational cost becomes very high. How to balance the time cost and the recognition rate is an open problem. In the final experiment, to further investigate the performance of the proposed method, we compare the recognition rate of our algorithm with two state-of-the-art methods, the Random + SRC method and the Downsample + SRC method [23]. In these two methods, the colour face images are treated using three separate channel components. We choose $L=4,5,6$ for all databases and a corresponding dimension $d=15,21,28$ from Eq. (39) for a sparse representation. From the Face94 database, we use only a subset made up of 1500 images from 153 subjects, with each subject providing 20 images. A total of 1200 $196 \times 196$ images are selected from the Face96 database to be used in the recognition experiment. All subjects from the Face95 and Grimace databases are used. From each of the colour face databases, we used 10 images of each subject as the training sample and used the remaining images as test samples. Table 5 shows the recognition rates for each method. The proposed method produces better recognition rates than the Random + SRC and Downsample + SRC methods.

\section{Conclusions}

In this paper, we first extend the conventional discrete Tchebichef moments defined for greyscale images to colour images using the quaternion algebra. The purpose is to 
process the colour image directly, without losing colour information. The main contributions of this paper are the following:

The quaternion Tchebichef moment invariants with respect to translation and scale transformations are constructed. The results obtained from real data show that the proposed descriptors are more robust to noise and have better descriptive capabilities than the existing methods.

A sparse-representation-based colour face recognition strategy using the proposed quaternion Tchebichef moments is introduced. The effectiveness of the classification method is demonstrated on colour face databases. The simulated results are compared with state-of-the-art methods. The experimental results demonstrate the advantages of our method over these well-known methods.

Future research will investigate the effectiveness of the proposed approach in other biometric verification applications and explore the optimal choice of quaternion Tchebichef moments of order $L$.

\section{Acknowledgements}

This work has been supported by the National Natural Science Foundation of China under Grant No. 61371150.

\section{References}

[1] R. Mukundan, S. H. Ong and P. A. Lee, "Image analysis by Tchebichef moments", IEEE Trans. Image Processing, vol. 10, (2001), pp. 1357-1364.

[2] X. Dai, H. Shu, L. Luo, G. N. Han and J. L. Coatrieuxc, "Reconstruction of tomographic images from limited range projections using discrete Radon transform and Tchebichef moments", Pattern Recognition, vol. 43, (2010), pp. 1152-1164.

[3] H. Zhu, H. Shu, T. Xia, L. M. Luo and J. L. Coatrieux, "Translation and scale invariants of Tchebichef moments, Pattern Recognition”, vol. 40, (2007), pp. 2530-2542.

[4] H. Zhang, X. Dai, P. Sun, H. Zhu and H. Shu, "Symmetric image recognition by Tchebichef moment invariants", Proceedings of 2010 IEEE 17th International Conference on Image Processing, (2010), September 26-29, Hong Kong

[5] Q. Liu, H. Zhu and Q. Li, "Image recognition by affine Tchebichef moment invariants", AICI 2011, Part III, LNAI 7004, (2011), pp. 472-480.

[6] Q. Li, H. Zhu and Q. Liu, "Image recognition by combined affine and blur Tchebichef moment invariants", 2011 4th International Congress on Image and Signal Processing, (2011), October 15-17, Shanghai, China.

[7] M. R. Teague, "Image analysis via the general theory of moments", J. Opt. Soc. Am., vol. 70, (1980), pp. 920-930.

[8] C. H. Teh and R. T. Chin, "On image analysis by the method of moments", IEEE Trans. Pattern Anal. Mach. Intell., vol. 10, (1988), pp. 496-513.

[9] Y. Sheng and L. Shen, "Orthogonal Fourier-Mellin moments for invariant pattern recognition”, J. Opt. Soc. Am. A, vol. 11, (1994), pp. 1748-1757.

[10] T. Suk and J. Flusser, "Affine moment invariants of color images", in: Proceedings of CAIP 2009, LNCS 5702, (2009), pp. 334-341.

[11] S. J. Sangwine, "Fourier transforms of colour images using quaternion or hypercomplex numbers", Electron, Lett., vol. 32, (1996), pp. 1979-1980.

[12] J. Vía, D. P. Palomar, L. Vielva and I. Santamaría, "Quaternion ICA from second-order statistics", IEEE Trans Signal Process., vol. 59, (2011), pp. 1586-1600.

[13] D. Assefa, L. Mansinha, K. F. Tiampo, H. Rasmussen and K. Abdella, "Local quaternion Fourier transform and color image texture analysis", Signal Processing, vol. 90, (2010), pp. 1825-1835.

[14] C. B. Gao, J. L. Zhou, J. R. Hu and F. N. Lang, "Edge detection of colour image based on quaternion fractional differential”, IET Image Process., vol. 5, (2011), pp. 261-272.

[15] B. C. Ujang, C. C. Took and D. P. Mandic, "Quaternion-valued nonlinear adaptive filtering”, IEEE Trans. Neural Networks, vol. 22, (2011), pp. 1193-1206.

[16] D. S. Alexiadis and G. D. Sergiadis, "Estimation of motions in color image sequences using hypercomplex Fourier transforms", IEEE Trans. Image Process., vol. 18, (2009), pp. 168-187. 
[17] J. Flusser and T. Suk, "Degraded image analysis: an invariant approach", IEEE Transactions on Pattern Analysis and Machine Intelligence, vol. 20, no. 6, (1998), pp. 590-603.

[18] Z. Chen and S.-K. Sun, "A Zernike moment phase-based descriptor for local image representation and matching”, IEEE Trans. Image Process., vol. 19, (2010), pp. 205-219.

[19] T. Suk and J. Flusser, "Affine moment invariants generated by graph method", Pattern Recognition, vol. 44, (2011), pp. 2047-2056.

[20] B. J. Chen, H. Z. Shu, H. Zhang, G. Chen, C. Toumoulin, J. L. Dillenseger and L. M. Luo, "Quaternion Zernike moments and their invariants for color image analysis and object recognition", Signal Processing, vol. 92, (2012), pp. 308-318.

[21] L.-Q. Guo and M. Zhu, "Quaternion Fourier-Mellin moments for color images”, Pattern Recogn., vol. 44, (2011), pp. 187-195.

[22] Mukundan, "Some computational aspects of discrete orthonormal moments", IEEE Trans. Image Process., vol. 13, (2004), pp. 1055-1059.

[23] J. Wright, A. Y. Yang, A. Ganesh, S. S. Sastry and Y. Ma, "Robust face recognition via sparse representation", IEEE Trans. Pattern Analysis and Machine Intelligence, vol. 31, (2009), pp. 210-227.

[24] S. H. Gao, I. W-H. Tsang and L-T. Chia, "Kernel sparse representation for image classification and face recognition", Proceedings of the 11th European Conference on Computer Vision, (2010), September 5-11, Crete, Greece.

[25] M. Yang and L. Zhang, "Gabor feature based sparse representation for face recognition with gabor occlusion dictionary", Proceedings of the 11th European Conference on Computer Vision, (2010), September 5-11, Crete, Greece.

[26] Y. Chen, T. T. Do and T. D. Tran, "Robust face recognition using locally adaptive sparse representation", Proceedings of 2010 IEEE 17th International Conference on Image Processing, (2010) September 26-29, Hong Kong

[27] M. Rajapakse, J. Tan and J. Rajapakse, "Color channel encoding with NMF for face recognition", Proceedings of 2004 IEEE 11th International Conference on Image Processing, (2004), October 24-27, Singapore.

[28] C. Z. Wang, B. C. Yin, X. M. Bai and Y. F. Sun, "Color face recognition based on 2DPCA", International Conference on Pattern Recognition, (2008), December 8-11, Florida, USA.

[29] Y. Sun, S. Chen and B. Yin, "Color face recognition based on quaternion matrix representation", Pattern Recognition Letters, vol. 32, (2011), pp. 597-605.

[30] W. R. Hamilton, "Elements of quaternions", London, U. K., Iongmans, Green (1866).

[31] C. E. Moxey, S. J. Sangwine and T. A. Ell, "Hypercomplex correlation techniques for vector images", IEEE Trans. Signal Process., vol. 51, (2003), pp. 1941-1953.

[32] T. A. Ell and S. J. Sangwine, "Hypercomplex Fourier transforms of color images", IEEE Trans. Image Process., vol. 16, (2007), pp. 22-35

[33] W. Feng and B. Hu, "Quaternion discrete Cosine transform and its application in color template matching", Congress on Image and Signal Processing, (2008), pp. 252-256.

[34] http://dces.essex.ac.uk/mv/allfaces/ 